\begin{tabular}{|c|c|}
\hline Title & $\begin{array}{l}\text { Consistent adjustment of radioactive decay and fission yields data with measurement data of decay heat and beta } \\
\text { delayed neutron activities }\end{array}$ \\
\hline Author(s) & Chiba, Go \\
\hline Citation & $\begin{array}{l}\text { A nnals of nuclear energy, 101, 23-30 } \\
\text { https://doi.org/10.1016j.anucene.2016.09.054 }\end{array}$ \\
\hline Issue Date & $2017-03$ \\
\hline Doc URL & http:/hdl. handle.net/2115/2704 \\
\hline Rights & $\begin{array}{l}\text { () 2017. This manuscript version is made available under the CC-BY-NC-ND } 4.0 \text { license } \\
\text { http://creativecommons.org/icenses/oy-nc-nd/4.0/ }\end{array}$ \\
\hline Rights(URL) & http://creativecommons.org/icenses/by-nc-nd/4.0/ \\
\hline Type & article (author version) \\
\hline File Information & main.pdf \\
\hline
\end{tabular}

Instructions for use 


\title{
Consistent adjustment of radioactive decay and fission yields data with measurement data of decay heat and $\beta$-delayed neutron activities
}

\author{
Go Chiba*,a \\ ${ }^{a}$ Hokkaido University, Kita-ku, Sapporo, Hokkaido 060-8628, Japan
}

\begin{abstract}
Decay heat and delayed neutron yields, which are important physical quantities in the field of the nuclear engineering, are dependent on common nuclear data such as radioactive decay data and fission yields data of fission product nuclides. In the present study, correlations between uncertainties of these two quantities are investigated.

Nuclear data relevant to uranium-235 and plutonium-239 fissions with thermal neutron are adjusted consistently with a procedure based on Bayes' theorem using the measurement data of decay heat and delayed neutron activities. Numerical results suggest that the correlation between decay heat and delayed neutron activities uncertainties is not significant, and that independent treatments of decay heat or delayed neutron activities are possible. The effect of the consistent treatment of decay heat and delayed neutron activities is, however, observed in the adjustment results in some nuclear data such as uranium-235 thermal fission yields of yttrium-100m and zirconium-100.

Key words: nuclear data adjustment, decay data, fission yields data, decay heat, $\beta$-delayed neutrons
\end{abstract}

\section{Introduction}

It is well known that the decay heat, which is accompanied with radioactive decay of unstable nuclides, are important in many aspects of the field of the nuclear engineering, such as nuclear power plant safety analyses and nuclear fuel waste management. The $\beta$-delayed (or delayed) neutron yields are also recognized important since they are vital to realize controllable fission chain reactions in nuclear fission reactors. Both of the decay heat and delayed neutron yields are dependent on common nuclear data which are time constants, paths, branching ratios and released energy of radioactive decay of fission product (FP) nuclides, and their fission yields. In conventional nuclear fission reactors analyses, both of them have been treated with simplified models such as polynomial fitting models for time-dependent decay heat calculations and six fictitious precursors models for delayed neutron yields. Although these simplified treatments have resulted

*Corresponding author, Tel: +81-10-706-6683, Fax: +81-10-706-6683

Email address: go_chiba@eng.hokudai.ac.jp (Go Chiba) 
in a great success in the field of the nuclear reactor engineering, further improvements are restricted since these treatments rely on limited experimental data. In addition, several advanced concepts of nuclear fission reactors adopt non-conventional nuclear fuels. For example, advanced fission reactors to burn minor actinides (MAs), such as accelerator-driven systems, employ MA-rich fuels. In such nuclear reactors, decay heat and delayed neutron yields of MA play important roles. Experimental data of MA nuclear data are, however, scarce, so it is difficult to derive reliable simplified models based on the measurement data.

Under such circumstances, approaches from the field of the nuclear physics have been considered important, and so much effort have been devoted to research and development activities, in which FP nuclides are explicitly treated in time-dependent decay heat or delayed neutron yields calculations.

Application of the summation calculations to actual problems requires accurate and reliable nuclear data of each FP nuclide. Sensitivity and uncertainty analyses are quite powerful to improve the fundamental nuclear data which are related to the target physical quantities, which are decay heat and delayed neutron activities in the present study. If one can quantify uncertainties of the target quantities and can specify the important nuclear data which are dominant contributors to total uncertainty, such information can be efficiently utilized by researchers working on the nuclear physics and the nuclear data.

Our research groups have worked on the uncertainty quantification of decay heat and delayed neutron yields independently so far(Chiba, 2015; Kawamoto, 2016). Since these two physical quantities are dependent on the same kind of the nuclear data as described above, consistent treatment is considered useful.

In the present study, we evaluate correlations between decay heat uncertainty and delayed neutron activities uncertainty for fission reactions of uranium-235 and plutonium-239 with thermal neutron, for which rich measurement data are avaliable. Nuclear data adjustment calculations based on Bayes' theorem are also conducted, and effectiveness of the consistent treatment of decay heat and delayed neutron activities is discussed.

The present paper is organized as follows; section 2 provides a brief description of fundamental theories of uncertainty propagation and nuclear data adjustment calculations. Sections 3 and 4 are devoted to describe numerical procedure and the used measurement data, respectively. Numerical results are presented in section 5, and conclusion of the present study and future perspective are described in section 6 .

\section{Theory}

In the present study, correlations between uncertainties of decay heat and delayed neutron activities after a burst fission are calculated. Uncertainty propagation calculations are performed with sensitivities of the target quantities with respect to the nuclear data of independent fission yields, decay constants, decay energy and decay branching ratios of FP nuclides, and the covariance data of these nuclear data. Nuclear data adjustment calculations using the measurement data are also carried out. In this section, we 
briefly describe theories of sensitivity calculations, uncertainty propagation calculations and nuclear data adjustment calculations.

\subsection{Sensitivity calculations with respect to nuclear data}

Decay heat and delayed neutron activity at time $t, H(t)$ and $Q(t)$, are represented with nuclide number densities and the relevant nuclear data as follows:

$$
\begin{aligned}
H(t) & =\sum_{i} E_{i} \lambda_{i} N_{i}(t), \\
Q(t) & =\sum_{i} \operatorname{Pn}_{i} \lambda_{i} N_{i}(t),
\end{aligned}
$$

where $E_{i}, N_{i}(t), \operatorname{Pn}_{i}$ and $\lambda_{i}$ are emitted energy per a decay, nuclide number density at $t$, neutron emission probability per decay and decay constant of nuclide $i$. In the present study, we consider decay heat and delayed neutron activities after a burst fission, so independent fission yields for a considered fissile nuclide are given as initial number densities.

Sensitivities of $H(t)$ and $Q(t)$ with respect to nuclear data $\sigma$ are written as $\frac{\partial H(t)}{\partial \sigma}$ and $\frac{\partial Q(t)}{\partial \sigma}$, where $\sigma$ can be $E_{j}, \lambda_{j}, \operatorname{Pn}_{j}$ and branching ratios. Since $N_{i}(t)$ is dependent on independent fission yields, decay constants and decay branching ratios, we have to calculate derivative of $N_{i}(t)$ with respect to these nuclear data in sensitivity calculations of $H(t)$ and $Q(t)$. This can be easily and efficiently carried out with the well-established generalized perturbation theory (GPT) for time-dependent problems(Lewins, 1960; Gandini, 1975). Since application of GPT to decay heat and delayed neutron activities calculations has been described in our previous papers in detail(Chiba, 2015; Kawamoto, 2016), it is omitted here.

\subsection{Uncertainty propagation calculations}

Let us denote the $i$ th target quantity as $R_{i}$, which is decay heat or delayed neutron activity at a certain time. Variance of $R_{i}, V_{R_{i}}$, can be calculated with the following well-known sandwich formula:

$$
V_{R_{i}}=\mathbf{S}_{R_{i}}^{T} \mathbf{V}_{\sigma} \mathbf{S}_{R_{i}}
$$

wh with respect to various nuclear data. The superscript $T$ denotes the transposition for matrices and vectors. Similarly, covariance between two quantities $R_{i}$ and $R_{j}, V_{R_{i}, R_{j}}$, can be calculated as follows:

$$
V_{R_{i}, R_{j}}=\mathbf{S}_{R_{i}}^{T} \mathbf{V}_{\sigma} \mathbf{S}_{R_{j}}
$$

and a covariance matrix for $I$ multiple quantities, $\mathbf{V}_{R}$, can be written as follows:

$$
\mathbf{V}_{R}=\mathbf{S}^{T} \mathbf{V}_{\sigma} \mathbf{S}
$$




\subsection{Nuclear data adjustment calculations}

If measurement data of decay heat or delayed neutron activities are available, these information can be efficiently used to modify probability distributions of the original nuclear data, and improvement on prediction accuracy of decay heat and delayed neutron activities is expected. This can be realized with the well-known nuclear data adjustment method(Dragt, 1977), which is based on Bayes' theorem.

Let us assume that $I$ measurement data of decay heat and delayed neutron activities are available, and they are denoted as $M_{i}(i=1,2, \ldots, I)$. It is also assumed that the covariance data of these measurement data are available and denoted as $\mathbf{V}_{M}$ in a matrix form. Numerical calculations with the original nuclear data give numerical results for each measurement data, and the numerical results are denoted as $C_{i}(i=$ $1,2, \ldots, I)$. Relative difference between numerical value and measurement data for the $i$ th measurement data, $D_{i}$, is defined as

$$
D_{i}=\left(C_{i}-M_{i}\right) / M_{i}=C_{i} / M_{i}-1, \quad(i=1,2, \ldots, I),
$$

and this is represented in a vector form as $\mathbf{D}$. With the nuclear data adjustment procedure, the original nuclear data $\sigma$ is revised as

$$
\tilde{\boldsymbol{\sigma}}=\boldsymbol{\sigma}+\mathbf{V}_{\sigma} \mathbf{S}\left(\mathbf{S}^{T} \mathbf{V}_{\sigma} \mathbf{S}+\mathbf{V}_{M}\right)^{-1} \mathbf{D},
$$

where $\tilde{\boldsymbol{\sigma}}$ is the revised (or adjusted) nuclear data through the adjustment. Note that uncertainty in numerical values due to numerical treatments is ignored here. The covariance matrix of the adjusted nuclear data, $\mathbf{V}_{\tilde{\sigma}}$, can be given as

$$
\mathbf{V}_{\tilde{\sigma}}=\mathbf{V}_{\sigma}-\mathbf{V}_{\sigma} \mathbf{S}\left(\mathbf{S}^{T} \mathbf{V}_{\sigma} \mathbf{S}+\mathbf{V}_{M}\right)^{-1} \mathbf{S}^{T} \mathbf{V}_{\sigma} .
$$

It is expected that the use of the adjusted nuclear data and their covariance matrix gives revised numerical results of decay heat and delayed neutron activities which are closer to the measurement data and which have smaller prediction uncertainties.

\section{Numerical calculation procedure}

The summation calculations for decay heat and delayed neutron activities after a burst fission require the relevant nuclear data and a numerical method to calculate time dependence of number densities of over 1,000 FP nuclides. In the present study, we utilize the revised versions of the JENDL Fission Product Decay Data File 2011 (JENDL/FPD-2011) and Fission Yields Data File 2011 (JENDL/FPY-2011)(Katakura, 2011, 2016). Number densities of all the nuclides for which the nuclear data are given in these files are traced with time in the numerical calculations. Number densities at time $t$ in a vector form is denoted as $\mathbf{N}(t)$, and this satisfies the following nuclide transmutation equation:

$$
\frac{d \mathbf{N}(t)}{d t}=\mathbf{A N}(t)
$$


where $\mathbf{A}$ is a nuclide transmutation matrix. Solution to Eq. (9) is represented with a matrix exponential as $\mathbf{N}(t)=\exp (\mathbf{A} t) \mathbf{N}(0)$. In the present study, this matrix exponential is numerically calculated with the Mini-Max Polynomial Approximation (MMPA) method(Kawamoto, 2015). Time-dependent decay heat and delayed neutron activities can be obtained from $\mathbf{N}(t)$ and the relevant nuclear data.

Sensitivities of decay heat and delayed neutron activities are calculated with the GPT for time-dependent problems. Adjoint number densities required for the sensitivity calculations are obtained by solving the adjoint nuclide transmutation equation with the matrix exponential method and the MMPA method.

In uncertainty propagation and nuclear data adjustment calculations, covariance data of relevant nuclear data are essential. We use the covariance data given in JENDL/FPY-2011 and JENDL/FPD-2011. Since covariance data are not given for some nuclear data in these files, we assume that these nuclear data have 100\% relative uncertainty like the previous study by Katakura(Katakura, 2013).

It has been widely recognized that there are strong negative correlations in independent fission yields among different nuclides belonging to a single mass chain, and these correlations have significant influence on uncertainty quantification calculations for decay heat and delayed neutron activities even though such correlation data are not provided in current evaluated nuclear data files. We apply a method of estimating covariances among different nuclides in a single mass chain, which has been proposed by Devillers(Devillers, 1977):

$$
\begin{aligned}
& \bar{V}_{i, i}=V_{i}\left(1-\frac{V_{i}}{V_{M Y}+\sum_{j=1}^{J} V_{j}}\right), \\
& \bar{V}_{i, j}=-\frac{V_{i} V_{j}}{V_{M Y}+\sum_{j=1}^{J} V_{j}},
\end{aligned}
$$

where $\bar{V}_{i, j}$ are covariance between fission yields of nuclides $i$ and $j, V_{i}$ is variance of fission yield of nuclide $i$ originally given in JENDL/FPY-2011, $V_{M Y}$ is variance of mass yield, and $J$ denotes the number of nuclides in the same mass chain.

Branching ratio uncertainties have also strong negative correlations among different decay paths of a single nuclide because of the normalization condition. This is also taken into consideration as our previous study(Chiba, 2015; Kawamoto, 2016).

\section{Measurement data}

In the present study, we will treat decay heat and delayed neutron activities after a burst fission of uranium-235 and plutonium-239 with thermal neutron. 
On the decay heat, we use the measurement data obtained at Oak Ridge National Laboratory (ORNL) by Dickens(Dickens, 1977, 1978) and the international fission decay standards data given by Tobias(Tobias, 1989). The former and latter data are referred to as 'ORNL' and 'Tobias' in the present paper. Standard deviations of each measurement data are also given in these references.

On the delayed neutron activities, we prepare fictitious measurement data from the six precursor-group data evaluated by Keepin(Keepin, 1957) since Keepin's evaluations were based on the measurement data. Time-dependent delayed neutron activities after a burst fission are calculated with Keepin's data, and neutron activities at 32 time points are regarded measurement values. Standard deviations of these fictitious measurement data are also calculated from uncertainty data of the six precursor-group data evaluated by Keepin.

\section{Numerical results}

\subsection{Comparison between numerical results and measurement data}

Figures 1 and 2 show comparisons between numerical (calculation) values and measurement data of $\gamma$ and $\beta$ components of decay heat after a burst fission of uranium-235 and plutonium-239 with thermal neutron. Error bars correspond to the standard deviation of the measurement data.

Figures 3 and 4 show nuclear data-wise uncertainties of $\gamma$ and $\beta$ components of decay heat after a burst fission of uranium-235 and plutonium-239 with thermal neutron. Whereas contributions of each nuclear data are similar between $\gamma$ and $\beta$ components of decay heat both in uranium-235 and plutonium-239 thermal fissions, significant differences are observed in nuclear data-wise contributions between uranium-235 and plutonium-239; while uncertainty of decay energy is the dominant contributor to the total uncertainty over entire time period in uranium-235 fission, uncertainty of fission yield is the dominant contributor during the period from $10^{3}$ to $10^{5}$ seconds in the $\gamma$ component of decay heat of plutonium-239 fission.

Figure 5 shows comparison between numerical values and the fictitious measurement data of delayed neutron activities after a burst fission of uranium-235 and plutonium-239 with thermal neutron. Error bars correspond to the standard deviation of the measurement data.

Figure 6 shows nuclear data-wise uncertainty of delayed neutron activities after a burst fission of uranium-235 and plutonium-239 with thermal neutron. It is interesting to point out that dominant contributors are different between uranium-235 and plutonium-239 thermal fissions, and that fission yields uncertainty, which is dominant contributor to the uncertainty of the $\gamma$ component of decay heat, is dominant over the entire time period in plutonium-239 fission.

In order to quantify the agreement between numerical values and measurement data and to check the consistency of the nuclear data and the measurement data with their covariance data, the following quantity 
of $\chi^{2}$ is calculated:

$$
\chi^{2}=\mathbf{D}^{T}\left(\mathbf{V}_{R}+\mathbf{V}_{M}\right)^{-1} \mathbf{D} .
$$

It is expected that $\chi^{2}$ divided by the degree of freedom (DOF), that is the number of observed quantities, is close to or less than unity if all the information including uncertainty are statistically consistent with each other. Table 1 shows the obtained $\chi^{2}$ over DOF. Since correlations of the decay heat measurement data uncertainty between different time points are not given in the references, three different values of correlations are assumed over the entire time period and $\chi^{2}$ over DOF is calculated for each assumed correlation. These correlations are given to each component of decay heat of each fissile isotope. Note that no correlations are assumed to the fictitious measurement data of the delayed neutron activities. On uranium-235 thermal fission, $\chi^{2}$ over DOF takes much larger values than unity when the correlation of 0.8 is assumed. On the other hand, statistically-consistent results are obtained on plutonium-239 thermal fission regardless with the assumption of the correlation. In the following calculations, correlation of 0.5 is assumed for the measurement data of decay heat.

\subsection{Nuclear data adjustment}

Nuclear data adjustment calculations are carried out using the numerical results obtained in the preceding section. The following three calculation cases are considered: only the measurement data of decay heat are used (case 1), only the delayed neutron activity data are used (case 2), and both the data are consistently used (case 3). Adjustment calculations of case 1 and case 2 are referred to as the independent adjustment in contrast with the consistent adjustment of case 3. Modifications in the nuclear data are calculated with Eq. (7), and revised numerical results of decay heat and delayed neutron activities are obtained with the adjusted nuclear data.

Figure 7 shows comparisons between numerical values, which are obtained with the adjusted nuclear data, and the measurement data of $\gamma$ and $\beta$ components of decay heat after a burst fission of uranium-235 with thermal neutron. It is difficult to distinguish the curve of the original nuclear data from that of case 2. This suggests that the delayed neutron activity data of uranium-235 thermal fission do not affect the decay heat after a burst fission at all. This is also supported by the fact that the curve of case 1 is almost identical to that of case 3 .

Figure 8 shows results on decay heat after a burst fission of plutonium-239 with thermal neutron. The curve of the original data is almost identical to that of case 2. It is interesting to point out that the curve of case 3 is different from that of case 1 around 10 seconds of cooling time. Consideration of delayed neutron activities data affects decay heat calculations through the nuclear data adjustment.

Figure 9 shows comparison between numerical values, which are obtained with the adjusted nuclear data, and the fictitious measurement data of delayed neutron activities after a burst fission of uranium-235 and plutonium-239 with thermal neutron. The curve of the original data is almost identical to that of case 
1 , but slight difference is observed in short time period after a burst fission of uranium-235. The curves of case 2 and case 3 agree with each other.

Figure 10 shows correlation matrices of nuclear data-induced uncertainties of decay heat and delayed neutron activities after a burst fission of uranium-235 and plutonium-239 with thermal neutron. The numbers in the $\mathrm{X}$ - and Y-axes denote the index of the integral quantities; the indices from 1 to 31 and those from 32 to 62 correspond to $\gamma$ and $\beta$ components of time-dependent decay heat, and those from 63 to 94 correspond to the time-dependent neutron activities. These quantities are ordered with time in each physical quantity (or property): $\gamma$ component of decay heat, $\beta$ component of decay heat and delayed neutron activities. Since these figures are shown to observe degree of correlations among different properties, absolute values of correlations are plotted. Figure 11 shows the same correlation matrix with a different range to enhance weak correlations among different properties. Generally, strong correlations are observed between two different cooling time close to each other in the same property. Correlations between different properties are generally small, but there exist correlations of approximately 0.4 between the $\gamma$ decay heat and $\beta$ decay heat. Among decay heat and delayed neutron activities, non-zero correlations are also observed. These correlations among different properties affect the nuclear data adjustment results described above.

Finally, modifications in fission yields through the nuclear data adjustment are listed in Table 2. These modifications are normalized by their standard deviations in the original nuclear data. In the most of the fission yields data, modifications by the consistent adjustment give the same results with the independent adjustment with the decay heat data or the delayed neutron activities data. However, on some fission yields data, such as yttrium-100m and zirconium-100 of uranium-235 thermal fission, modifications by the consistent adjustment give different results with the independent adjustments.

\section{Concluding remarks}

Decay heat and delayed neutron yields, which are important physical quantities in the field of the nuclear engineering, are dependent on common nuclear data such as decay data and fission yields data of fission product nuclides. In the present study, correlations between uncertainties of these two quantities have been investigated.

Nuclear data relevant to uranium-235 and plutonium-239 fissions with thermal neutron have been adjusted consistently with a procedure based on Bayes' theorem using the measurement data of decay heat and delayed neutron activities. Numerical results have suggested that the correlation between decay heat and delayed neutron activities uncertainties is not significant, and that independent treatments of decay heat or delayed neutron activities are possible. The effect of the consistent treatment of decay heat and delayed neutron activities, however, has been observed in the adjustment results in some nuclear data such as uranium-235 thermal fission yields of yttrium-100m and zirconium-100. 
Extensive consistent-nuclear data adjustment calculations for various fission energy, fissile nuclides and irradiation conditions are planned as a future subject.

\section{References}

Chiba, G., Narabayashi, T., 2015. Uncertainty quantification of total delayed neutron yields and time-dependent delayed neutron emission rates in frame of summation calculations, Ann. Nucl. Energy, 85, 846-855.

Devillers, C., 1977. The importance of fission product nuclear data in reactor design and operation, IAEA Panel, Petten.

Dickens, J.K., Emery, J.F., Love, T.A., McConnell, J.W., Northcutt, K.J., Peelle, R.W., Weaver, H., 1977. Fission product energy release for time following thermal neutron fission of ${ }^{235} \mathrm{U}$ between 2 and 14,000 seconds, ORNL/NUREG-14, Oak Ridge National Laboratory.

Dickens, J.K., Emery, J.F., Love, T.A., McConnell, J.W., Northcutt, K.J., Peelle, R.W., Weaver, H., 1978. Fission product energy release for time following thermal neutron fission of ${ }^{239} \mathrm{Pu}$ between 2 and 14,000 seconds, ORNL/NUREG-34, Oak Ridge National Laboratory.

Dragt, J.B., Dekker, J.W.M., Gruppelaar, H., Janssen, A.J., 1977. Method of adjustment and error evaluation of neutron capture cross sections; application to fission product nuclides, Nucl. Sci. Eng., 62, 117-129.

Gandini, A., 1975. Time-dependent generalized perturbation theory for burn-up analysis, CNEN RT/FI(75)4, Comitato Nazionale per l'Energia Nucleare.

Katakura, J., 2011. JENDL FP decay data file 2011 and fission yields data file 2011, JAEA-Data/Code 2011-025, Japan Atomic Energy Agency.

Katakura, J., 2013. Uncertainty analyses of decay heat summation calculations using JENDL, JEFF and ENDF files, J. Nucl. Sci. Technol., 50, 799-807.

Katakura, J., Minato, F., Ohgama, K., 2016. Revision of the JENDL FP Fission Yield Data, EPJ Web of Conferences 111, 08004.

Kawamoto, Y., Chiba, G., Tsuji, M., Narabayashi, T., 2015. Numerical solution of matrix exponential in burn-up equation using mini-max polynomial approximation, Ann. Nucl. Energy, 80, 219-224.

Kawamoto, Y., Chiba, G., 2016. Feasibility study of decay heat uncertainty reduction using nuclear data adjustment method with experimental data, J. Nucl. Sci. Technol., (accepted).

Keepin, G.R., Wimett, T.F., Ziegler's, R.K., 1957. Delayed neutrons from fissionable isotopes of uranium, plutonium, and thorium, Physical Review, 107, 1044-1049.

Lewins, J., 1960. The time-dependent importance of neutrons and precursors, Nucl. Sci. Eng., 7, $268-274$.

Tobias, A., 1989. Derivation of decay heat benchmarks for U235 and Pu239 by a least square fit to measured data, Technical report, Central Electricity Generating Board. 
Table 1: $\chi^{2}$ over degree of freedom in (C/M-1) normalized by uncertainties for decay heat and delayed neutron activity

(a) U-235

\begin{tabular}{ccccc}
\hline Data & DOF & \multicolumn{3}{c}{ Correlation* } \\
& & 0.8 & 0.5 & 0.2 \\
\hline Tobias- $\gamma$ & 31 & 2.89 & 1.72 & 1.36 \\
Tobias- $\beta$ & 31 & 0.75 & 0.37 & 0.25 \\
ORNL- $\gamma$ & 32 & 3.81 & 2.06 & 1.93 \\
ORNL- $\beta$ & 32 & 0.52 & 0.24 & 0.18 \\
\hline Tobias & 62 & 1.80 & 1.03 & 0.80 \\
ORNL & 64 & 2.17 & 1.16 & 1.06 \\
\hline Tobias+ORNL & 126 & 3.04 & 1.45 & 1.16 \\
\hline Tobias+ORNL+Delayed neutron activitiy data & 158 & 2.54 & 1.27 & 1.04 \\
\hline
\end{tabular}

(b) $\mathrm{Pu}-239$

\begin{tabular}{ccccc}
\hline Data & DOF & \multicolumn{3}{c}{ Correlation* } \\
& & 0.8 & 0.5 & 0.2 \\
\hline Tobias- $\gamma$ & 31 & 1.15 & 0.68 & 0.52 \\
Tobias- $\beta$ & 31 & 0.58 & 0.28 & 0.20 \\
ORNL- $\gamma$ & 31 & 0.29 & 0.19 & 0.16 \\
ORNL- $\beta$ & 31 & 0.24 & 0.18 & 0.20 \\
\hline Tobias & 62 & 0.88 & 0.49 & 0.37 \\
ORNL & 62 & 0.27 & 0.19 & 0.18 \\
\hline Tobias+ORNL & 124 & 0.93 & 0.45 & 0.33 \\
\hline Tobias+ORNL+Delayed neutron activity data & 156 & 0.86 & 0.49 & 0.39 \\
\hline
\end{tabular}

* Correlation among measurement data of decay heat in the same time sequence 
Table 2: Modifications in fission yields through the nuclear data adjustment normalized by standard deviations

(a) U-235

\begin{tabular}{cccc}
\hline Nuclide & Case 1 & Case 2 & Case 3 \\
\hline Y-100m & +0.33 & -0.64 & -0.30 \\
Zr-100 & -0.31 & +0.56 & +0.24 \\
Sn-130 & +1.07 & +0.00 & +1.08 \\
Sn-130m & -1.19 & +0.02 & -1.18 \\
Sb-130m & +0.71 & +0.00 & +0.71 \\
Sb-137 & +0.00 & -1.00 & -1.06 \\
I-134m & +0.78 & -0.01 & +0.80 \\
I-137 & +0.00 & +0.93 & +0.95 \\
\hline
\end{tabular}

(b) $\mathrm{Pu}-239$

\begin{tabular}{cccc}
\hline Nuclide & Case 1 & Case 2 & Case 3 \\
\hline As-85 & +0.02 & -0.50 & -0.49 \\
Br-90 & +0.01 & -0.54 & -0.53 \\
Rb-94 & +0.03 & -1.00 & -0.96 \\
Sr-94 & -0.01 & +0.61 & +0.60 \\
Y-98m & +0.01 & -1.66 & -1.59 \\
Y-100m & -0.03 & -0.53 & -0.56 \\
Zr-98 & -0.01 & +1.53 & +1.44 \\
Sb-129m & +0.61 & +0.00 & +0.61 \\
Sb-135 & -0.06 & -0.83 & -0.90 \\
Te-134 & -0.51 & +0.00 & -0.51 \\
Te-135 & -1.47 & +0.04 & -1.39 \\
Te-136 & -0.06 & -0.46 & -0.50 \\
Te-137 & +0.01 & -0.61 & -0.59 \\
I-134m & +0.63 & +0.01 & +0.64 \\
I-135 & -0.58 & +0.02 & -0.55 \\
I-137 & -0.00 & -0.70 & -0.70 \\
I-138 & +0.09 & -0.55 & -0.46 \\
I-139 & +0.03 & -0.70 & -0.67 \\
Xe-135m & +1.87 & +0.04 & +1.87 \\
Xe-137 & -0.01 & +0.76 & +0.75 \\
\hline & & & \\
\hline
\end{tabular}


(a) $\gamma$

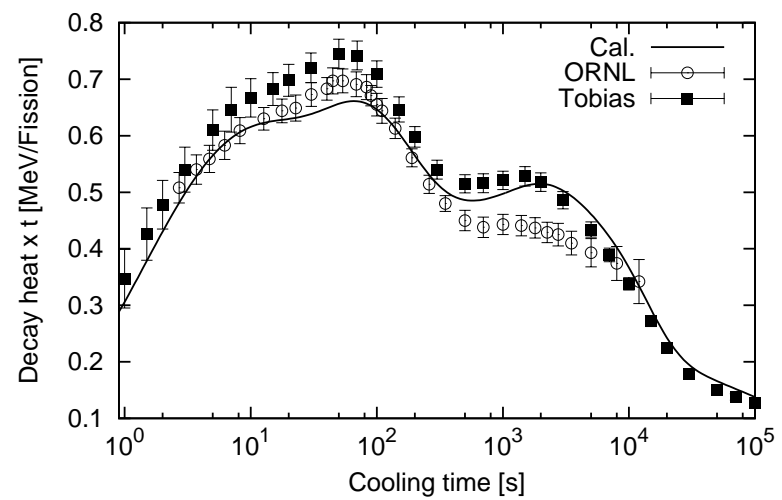

(a) $\beta$

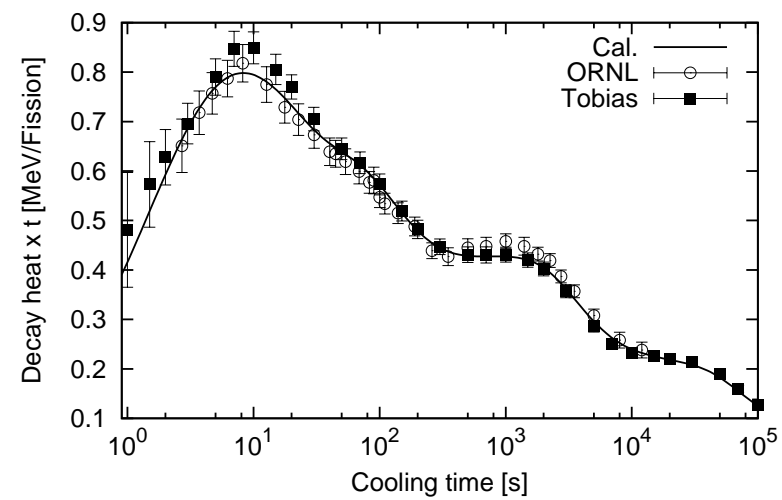

Figure 1: Comparisons between numerical values and measurement data of decay heat after a burst fission of uranium-235 with thermal neutron 
(a) $\gamma$

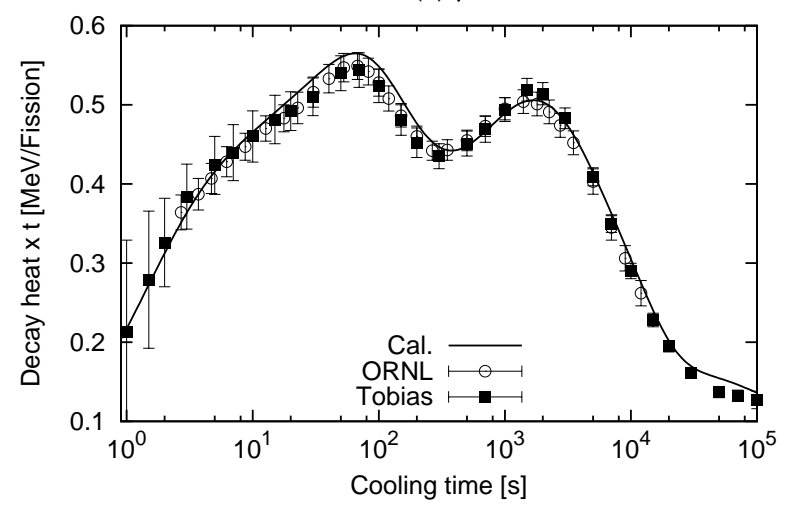

(b) $\beta$

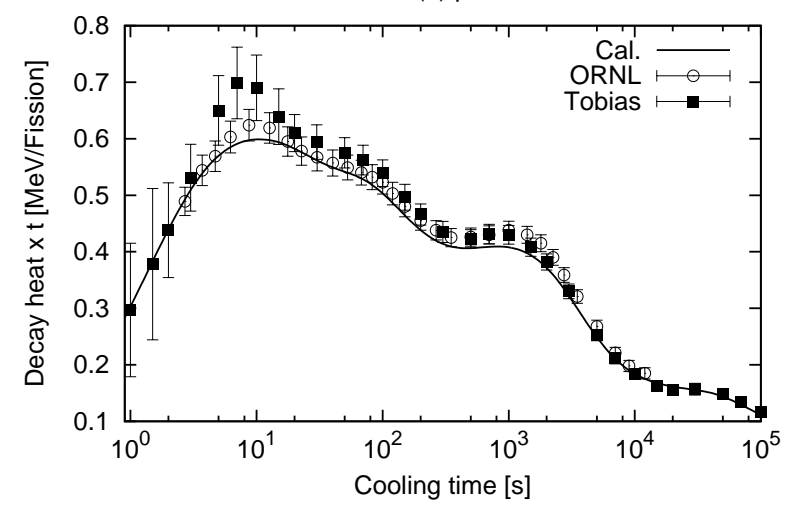

Figure 2: Comparisons between numerical values and measurement data of decay heat after a burst fission of plutonium-239 with thermal neutron 
(a) $\gamma$

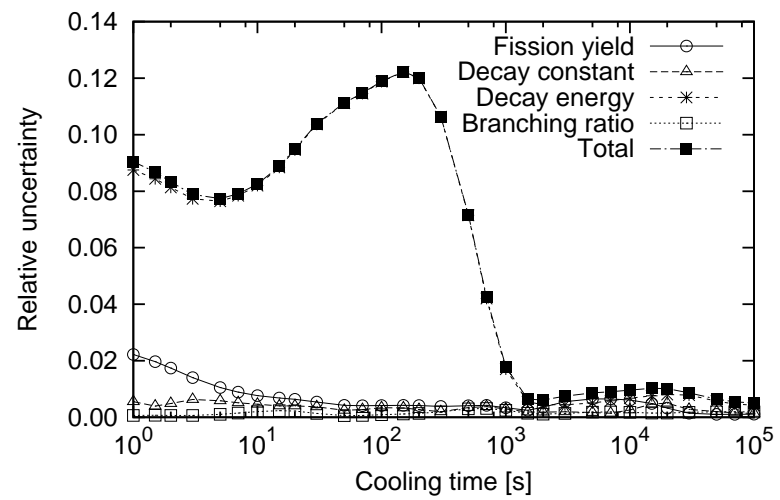

(b) $\beta$

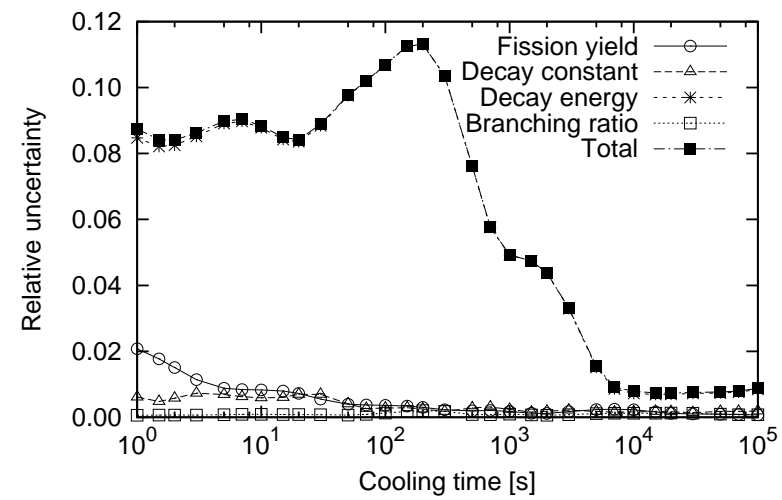

Figure 3: Nuclear data-wise uncertainties of decay heat after a burst fission of uranium-235 with thermal neutron 
(a) $\gamma$

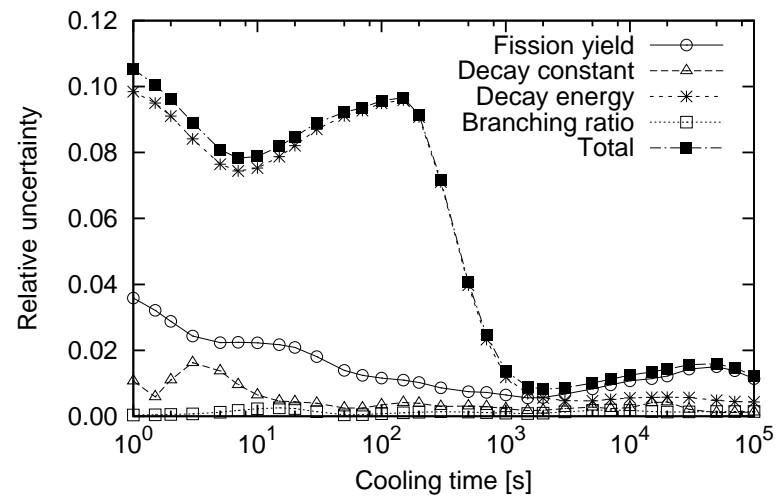

(b) $\beta$

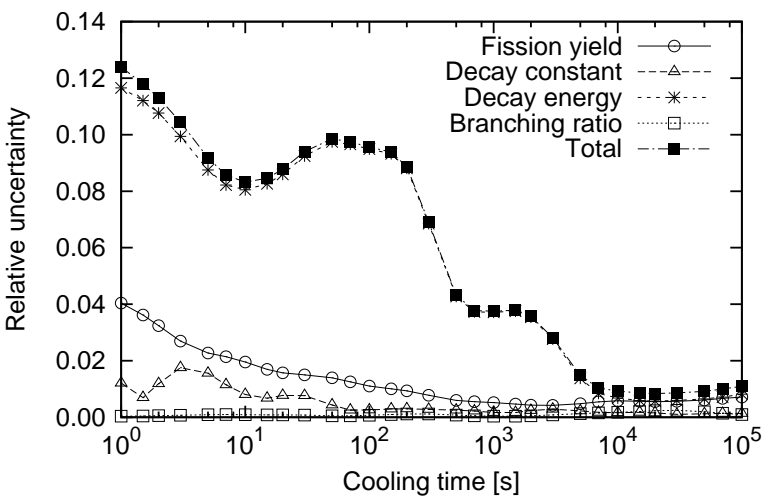

Figure 4: Nuclear data-wise uncertainties of decay heat after a burst fission of plutonium-239 with thermal neutron 
(a) U-235

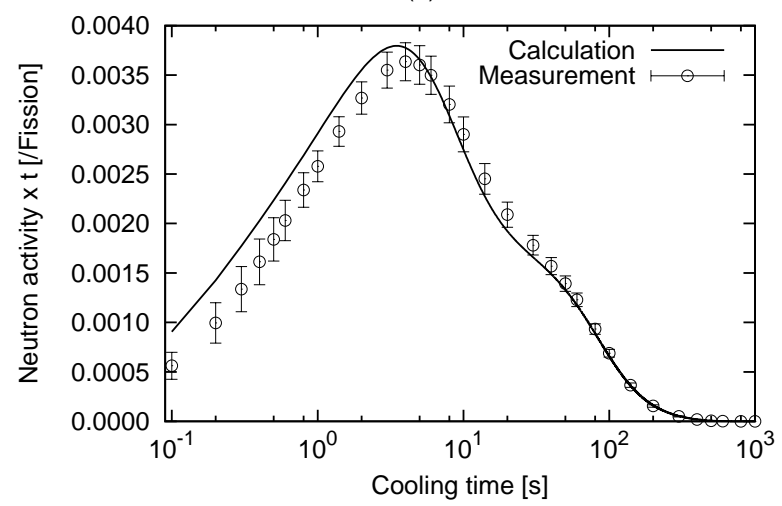

(b) Pu-239

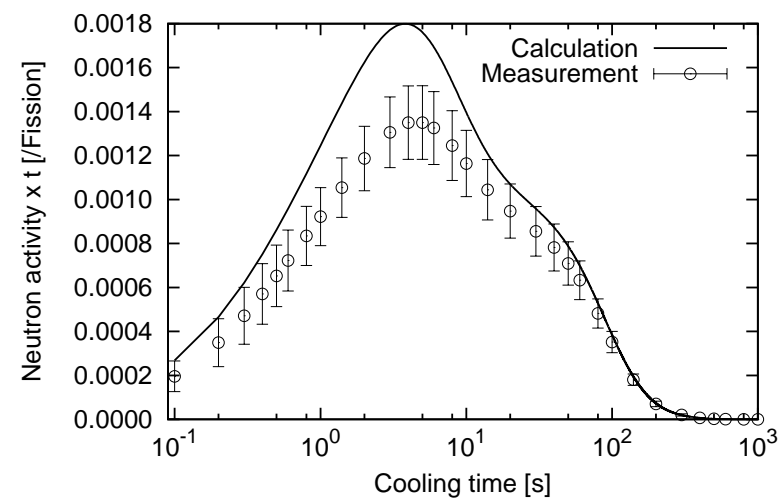

Figure 5: Comparisons between numerical values and measurement data of neutron activities after a burst fission with thermal neutron 
(a) U-235

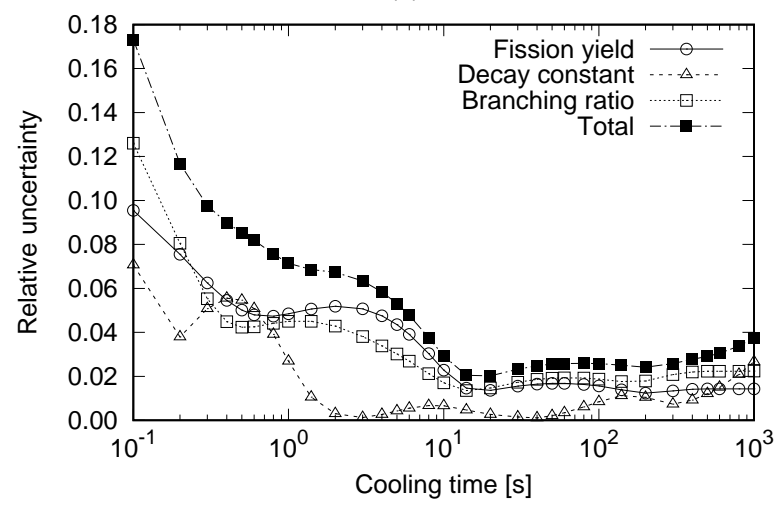

(b) Pu-239

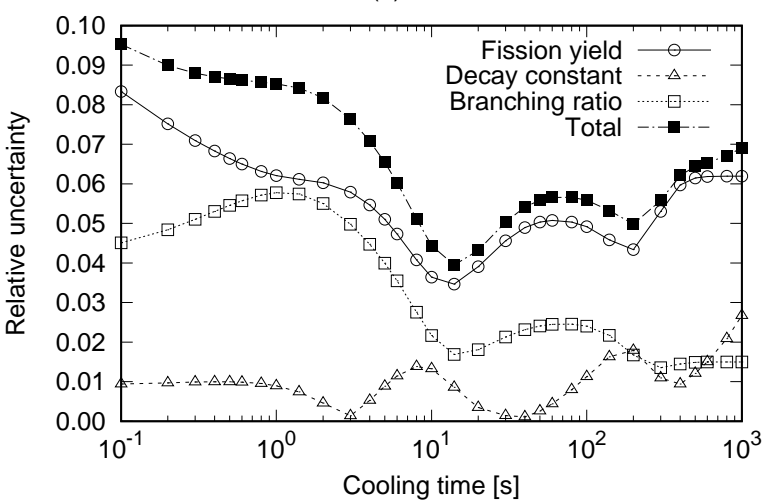

Figure 6: Nuclear data-wise uncertainties of neutron activities after a burst fission with thermal neutron 
(a) $\gamma$

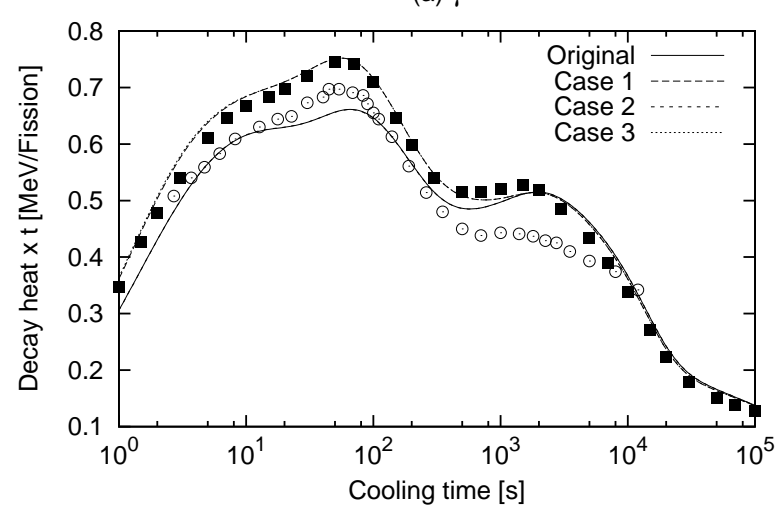

(b) $\beta$

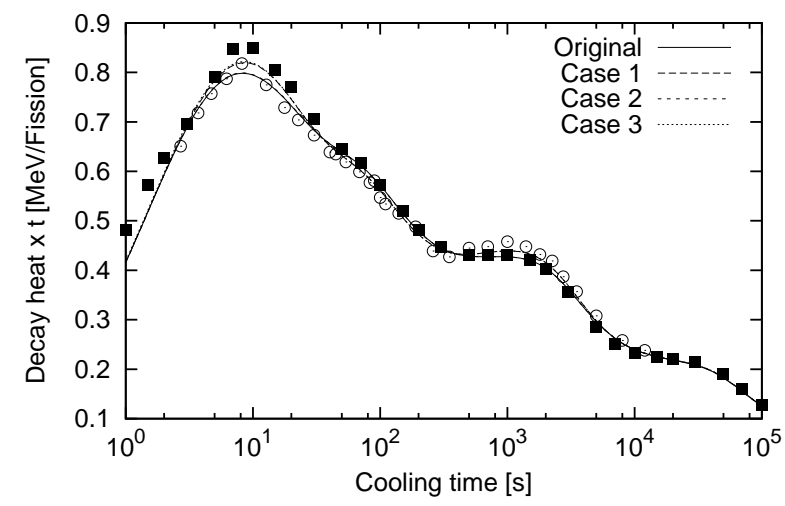

Figure 7: Comparisons between numerical values and measurement data of decay heat after a burst fission of uranium-235 with thermal neutron after nuclear data adjustment 
(a) $\gamma$

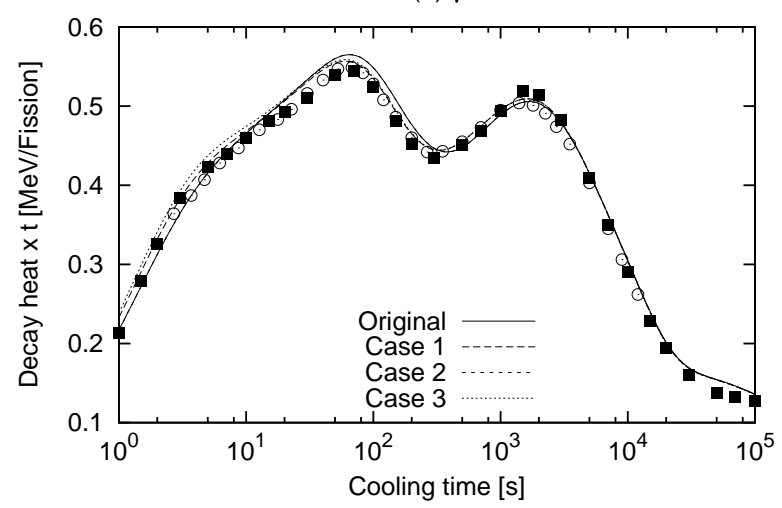

(b) $\beta$

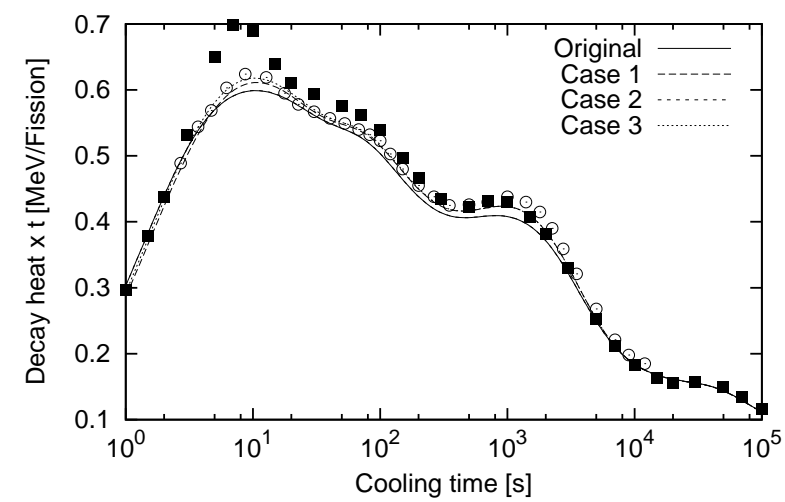

Figure 8: Comparisons between numerical values and measurement data of decay heat after a burst fission of plutonium-239 with thermal neutron after nuclear data adjustment 
(a) U-235

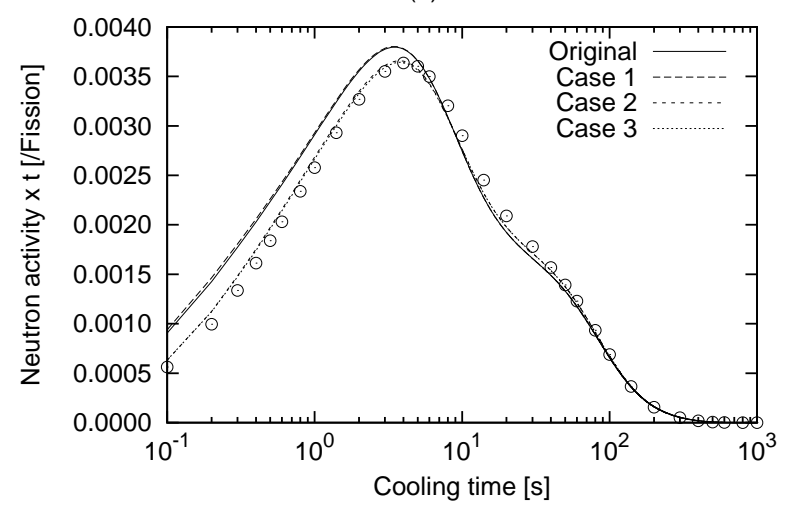

(b) Pu-239

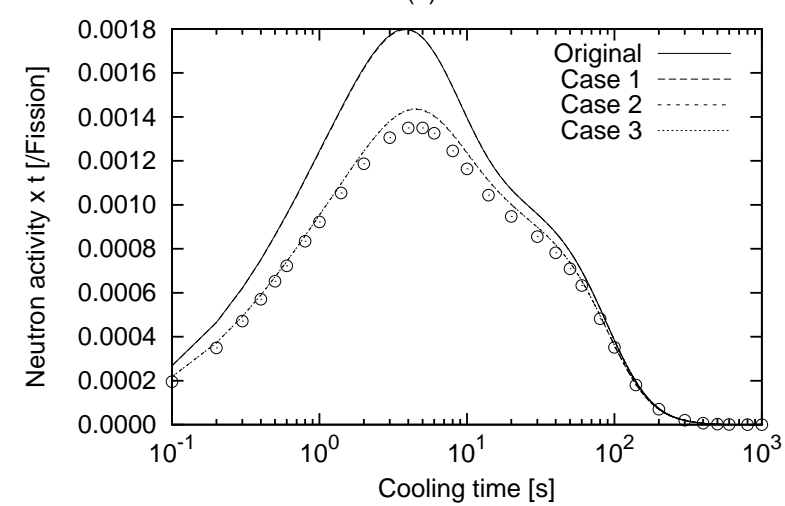

Figure 9: Comparisons between numerical values and measurement data of neutron activities after a burst fission with thermal neutron after nuclear data adjustment 


$$
\text { ヘル }
$$


(a) U-235

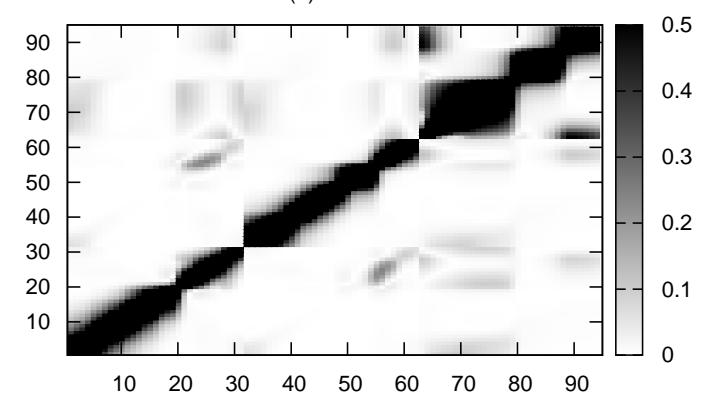

(b) Pu-239

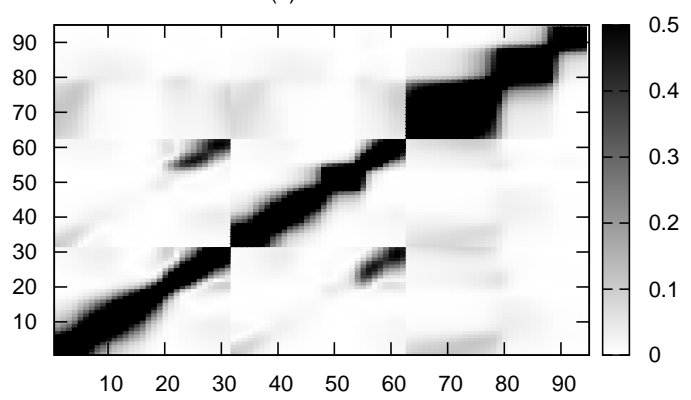

Figure 11: Absolute correlation matrices of nuclear data-induced uncertainty of decay heat and delayed neutron activities in a limited range 


\section{List of Figure Captions}

Fig.1 Comparisons between numerical values and measurement data of decay heat after a burst fission of uranium-235 with thermal neutron

Fig.2 Comparisons between numerical values and measurement data of decay heat after a burst fission of plutonium-239 with thermal neutron

Fig.3 Nuclear data-wise uncertainties of decay heat after a burst fission of uranium-235 with thermal neutron

Fig.4 Nuclear data-wise uncertainties of decay heat after a burst fission of plutonium-239 with thermal neutron

Fig.5 Comparisons between numerical values and measurement data of neutron activities after a burst fission with thermal neutron

Fig.6 Nuclear data-wise uncertainties of neutron activities after a burst fission with thermal neutron

Fig.7 Comparisons between numerical values and measurement data of decay heat after a burst fission of uranium-235 with thermal neutron after nuclear data adjustment

Fig.8 Comparisons between numerical values and measurement data of decay heat after a burst fission of plutonium-239 with thermal neutron after nuclear data adjustment

Fig.9 Comparisons between numerical values and measurement data of neutron activities after a burst fission with thermal neutron after nuclear data adjustment

Fig.10 Absolute correlation matrices of nuclear data-induced uncertainty of decay heat and delayed neutron activities

Fig.11 Absolute correlation matrices of nuclear data-induced uncertainty of decay heat and delayed neutron activities in a limited range 